\title{
O uso de software na assistência de enfermagem - Revisão de literatura
}

\author{
The use of software in nursing care - Literature review \\ El uso de software en la atención de enfermería - Revisión de la literatura
}

Danielle Freire Goncalves

ORCID: https://orcid.org/0000-0002-2469-1876

Universidade do Estado do Pará, Brasil

E-mail: adaniellefreire@gmail.com

Fabiana Morbach da Silva

ORCID: https://orcid.org/0000-0003-1583-0480 Universidade do Estado do Pará, Brasil

E-mail: Fabimorbach@gmail.com

Thiago Simplício Costa

ORCID: https://orcid.org/0000-0002-6349-7013 Universidade do Estado do Pará, Brasil E-mail: thiagosimplicio221@gmail.com

Iasmim Ianne Sousa Tavares

ORCID: https://orcid.org/0000-0003-2350-874X Faculdade de Ensino Superior da Amazônia Reunida, Brasil E-mail: iasmim.tavares14@gmail.com

Amanda Louise Rodrigues

ORCID: https://orcid.org/0000-0003-2399-7512 Universidade do Estado do Pará, Brasil E-mail: oliveira_louise12@hotmail.com

Amanda Gomes Diniz Pimenta

ORCID: https://orcid.org/0000-0001-7098-7224 Universidade do Estado do Pará, Brasil

E-mail: amandagdpepper@gmail

Adriana Maria Pantoja Malato

ORCID: https://orcid.org/0000-0002-3147-9953 Universidade Federal do Pará, Brasil

E-mail: adrimalato00@gmail.com

Ana Luisa Lemos Bezerra

ORCID: https://orcid.org/0000-0001-7111-3879 Universidade do Estado do Pará, Brasil

E-mail: 1998analuisa@gmail.com

Alessandro Souza Silva

ORCID: https://orcid.org/0000-0003-2745-9412 Universidade do Estado do Pará, Brasil E-mail: as-souzasilva2010@bol.com.br

Joelson Rodrigues Farias

ORCID: https://orcid.org/0000-0001-8890-2209 Universidade Federal do Pará, Brasil

E-mail: joelsonrodrigues1309@gmail.com

João Paulo Nascimento dos Anjos ORCID: https://orcid.org/0000-0001-6479-4610 Universidade do Estado do Pará, Brasil

E-mail: jpnascimentodosanjos@gmail.com Savio da Silva de Figueiredo

ORCID: https://orcid.org/0000-0002-1362-2453 Universidade do Estado do Pará, Brasil E-mail: figueiredosavio09@gmail.com

Paulo Henrique de Melo Ferreira ORCID: https://orcid.org/0000-0002-0426-1027 Universidade do Estado do Pará, Brasil

E-mail: contato.pauloferreira@outlook.com Vanessa Ellen Matias Batista

ORCID: https://orcid.org/ 0000-0002-3859-5332 Universidade do Estado do Pará, Brasil E-mail: vaneellenmatias@gmail.com

Pedro Lucas Carrera da Silva

ORCID: https://orcid.org/0000-0002-7728-8554 Universidade do Estado do Pará, Brasil E-mail: pedrolucas02890@gmail.com 


\title{
Resumo
}

Objetivo: este artigo tem como objetivo analisar o banco de dados disponíveis sobre o uso de software na assistência de enfermagem, assim catalogar e disponibilizar uma lista dos artigos voltados para essa atualização do fazer saúde. Metodologia: trata-se de uma revisão de literatura, sendo assim, foi realizado uma busca nas bases de dados periódicos capes e LILACS, publicados nos últimos cinco anos e com relevância bibliográfica. Além disso, foram utilizados os Descritores em Ciências da Saúde (DECS): Software; Assistência de enfermagem; Inovação. Resultados: foram encontrados artigos científicos voltados para o assistencialismo e modernização do trabalho da enfermagem, demonstrando que o uso desse novo modo de se fazer saúde está na realidade da equipe de enfermagem, sendo assim, há a necessidade dessa equipe de acompanhar o ritmo de evolução de acordo com as necessidades do mercado de trabalho. Considerações finais: é indubitavelmente necessária uma maior publicação de estudos na área, para que os profissionais tenham mais embasamento científico sobre as transformações no campo da saúde, além de um maior interesse por parte da equipe de enfermagem em atualizar o modo de se fazer saúde.

Palavras-chave: Software; Assistência de enfermagem; Inovação.

\begin{abstract}
Objective: this article aims to analyze the available database on the use of software in nursing care, as well as cataloging and making available a list of articles aimed at this update on doing health. Methodology: this is a review of the literature, therefore, a search was performed in the periodical databases CAPES and LILACS, published in the last five years and with bibliographic information. In addition, Health Sciences Descriptors (DECS) were used: Softwares; Nursing care; Innovation. Results: scientific articles were found focused on assistentialism and modernization of nursing work, demonstrating that the use of this new way of doing health is in the reality of the nursing team, therefore, there is a need for this team to monitor the pace of evolution according to the needs of the labor market. Final considerations: a greater publication of studies in the area is undoubtedly necessary, so that professionals have more scientific basis on the transformations in the health field, in addition to a greater interest on the part of the nursing team in updating the way of doing health.
\end{abstract}

Keywords: Software; Nursing care; Innovation.

\section{Resumen}

Objetivo: este artículo tiene como objetivo analizar la base de datos disponible sobre el uso de software en el cuidado de enfermería, así como catalogar y poner a disposición una lista de artículos destinados a esta actualización sobre hacer salud. Metodología: se trata de una revisión de la literatura, por lo que se realizó una búsqueda en las bases de datos periódicas CAPES y LILACS, publicadas en los últimos cinco años y con información bibliográfica. Además, se utilizaron Descriptores de Ciencias de la Salud (DECS): Softwares; Cuidado de enfermera; Innovación. Resultados: se encontraron artículos científicos enfocados en asistencialismo y modernización del trabajo de enfermería, demostrando que el uso de esta nueva forma de hacer salud está en la realidad del equipo de enfermería, por lo tanto, existe la necesidad de que este equipo monitoree el ritmo de evolución. según las necesidades del mercado laboral. Consideraciones finales: sin duda es necesario publicar más estudios en el área, para que los profesionales tengan más bases científicas sobre las transformaciones en el campo de la salud, además de un mayor interés por parte del equipo de enfermería en actualizar la forma de hacer salud.

Palabras clave: Software; Cuidado de enfermera; Innovación.

\section{Introdução}

A enfermagem por muitos anos foi descrita como a arte do cuidado, entretanto, com o passar dos anos a enfermagem se desenvolveu e hoje ela é reconhecida como uma ciência, sempre procurando novos caminhos para se fazer uma saúde humanizada e acolhedora. Como toda ciência, e assim como todas as profissões na atualidade, a enfermagem vem se aprimorando e dinamizando com o passar do tempo ao inserir-se nas novas tecnologias (Acauan et al., 2020; Goncalves, 2020; Friedrich, 2019).

O modo de se fazer saúde vem se atualizando cada vez mais e o uso de tecnologias nesse meio vem crescendo de maneira exponencial, bem como auxiliando no acompanhamento da saúde dos indivíduos de maneira personalizada e efetiva. Com isso, os profissionais da saúde têm que se atualizar sobre as novas formas de prestação de cuidados aos pacientes, além de compreender a importância do uso de novas tecnologias para o benefício e favorecimento das informações melhorando a qualidade do cuidado prestado a população. De outro modo, a gestão de dados para os enfermeiros é otimizada com o uso de softwares, tornando-a, assim, um trabalho mais limpo e prático, tanto no meio nacional como no meio internacional (Carlantonio, 2016; Mota et al., 2019; Santos et al., 2020). 
Sabe-se que a enfermagem tem papel decisivo e proativo no cuidado ao paciente e a prestação desse serviço de enfermagem é baseada na comunicação clara do profissional com o paciente, por isso é imprescindível que se crie meio que facilite essa relação. Com isso, a enfermagem vê a necessidade de criar tecnologias para melhorar a comunicação, assimilação e diálogo da equipe e, assim, fortalecer essas relações para promover a saúde (Costa et al., 2019; Machado \& Vieira, 2020; Torres, 2018).

Aplicativos móveis estão presentes na vida da maioria da população, sendo assim, pode-se utilizá-los como aliados na prestação de cuidados, principalmente pela enfermagem que está presente a todo momento no desenvolvimento e prestação de cuidados à comunidade. Formas inovadoras de se fazer saúde não só facilitam o cuidado para os profissionais, como também desenvolvem uma saúde mais humanizada e empática, se utilizada de maneira amorosa, acolhedora e qualificada, além de aprimorar o processo de ensino-aprendizagem (Torres, 2018; Mota et al., 2019).

A equipe de enfermagem tem como uma das principais funções a educação em saúde para todos as faixas etárias e grupos sociais, com isso, um meio encontrado para alcançar os jovens foi a introdução de softwares para incluir a promoção da saúde, protagonizando mudanças nos hábitos que tem como resultado uma melhor qualidade de vida e uma redução no adoecimento da população. Reduzindo assim o tempo de espera para atendimentos, tanto no Sistema Único de Saúde como na rede privada, tornado a saúde mais eficaz e otimizada (Lima, 2019).

Ademais, o mercado de trabalho necessita de profissionais da saúde habilitados não só para utilizar as novas tecnologias emergentes, mas como também, capazes de desenvolvê-las. Pois, é o profissional que está inserido nesse ambiente é que reconhece as reais necessidades da saúde e o que deve ser feito para melhorar a qualidade de vida não só de pessoas não-saudáveis como também das pessoas saudáveis para prevenir possíveis casos de adoecimento.

\section{Metodologia}

Trata-se uma revisão integrativa da literatura, constituída de etapas: definição do tema; elaboração da pergunta norteadora; escolha dos critérios de inclusão e exclusão dos artigos; análise dos resumos das produções científicas, com foco nos objetivos; e por fim, análise dos dados. Buscou-se responder à questão norteadora: Quais são os softwares utilizados para auxiliar a assistência de enfermagem nos últimos 6 anos?

Para isso, foram utilizados levantamento bibliográficos no período de 2016 a 2021 nas plataformas Periódicos Capes e LILACS, tendo como critérios de inclusão artigos completos, disponíveis de forma gratuita em meio eletrônico e em português, bem como os artigos repetidos foram excluídos. Os Descritores em Ciências da Saúde (DECS) foram: Software; Assistência de enfermagem; Inovação. Ademais, para suporte do presente trabalho, a metodologia baseou-se na produção de Pereira, A. S. et al (2018).

Para seleção dos estudos, foi realizada uma leitura minuciosa dos títulos e dos resumos, a fim de verificar quais se encaixavam na questão norteadora e nos critérios de inclusão e exclusão.

\section{Resultados e Discussão}

A quantidade de estudos encontrados combinando os descritores citados anteriormente totalizou 401 artigos. Após essa pesquisa, os critérios de inclusão e exclusão foram aplicados, conforme descritos abaixo no Quadro 1, até chegar a amostragem final desta revisão integrativa, a qual totalizou 10 produções científicas. 
Quadro 1 - Aplicação dos critérios de inclusão e exclusão da presente revisão de literatura.

\begin{tabular}{|l|l|l|}
\hline Base de Dados & Periódicos CAPES & LILACS \\
\hline Descritores utilizados para pesquisa & $\begin{array}{l}\text { Software } \\
\text { - Assistência de } \\
\text { Enfermagem }\end{array}$ & $\begin{array}{l}\text { - Software } \\
\text { Enfermagem } \\
\text { - Inovação }\end{array}$ \\
\hline Quantidade de estudos encontrados a partir dos descritores & 397 & 4 \\
\hline $\begin{array}{l}\text { Quantidade de estudos que permaneceram após aplicação dos filtros de intervalo } \\
\text { dos últimos 5 anos }\end{array}$ & 236 & 4 \\
\hline Quantidade de estudos excluídos por se repetirem em diferentes bases ou idioma & 86 & 2 \\
\hline Quantidade de estudos excluídos por não corresponderem a temática proposta & 142 & 0 \\
\hline Total de estudos selecionados & 8 & 2 \\
\hline
\end{tabular}

Fonte: Autores (2021).

As 10 produções científicas escolhidas foram descritas nos Quadros 2 e 3, de modo que foram encontrados na íntegra no Portal de Periódicos CAPES, o qual compila diversas bases de dados, bem como na base de dados da Literatura LatinoAmericana e do Caribe em Ciências da Saúde (LILACS). A amostragem final constituiu-se de estudos em português publicados em periódicos brasileiros no intervalo de 2017 a 2021.

Quadro 2 - Distribuição dos artigos científicos selecionados em número, autor, título e ano.

\begin{tabular}{|c|c|c|c|}
\hline $\begin{array}{l}\mathbf{N}^{\circ} \quad \text { do } \\
\text { Artigo }\end{array}$ & Autores & Título & Ano \\
\hline 1 & $\begin{array}{l}\text { DiCarlantonio LFM, Freitas LCM, } \\
\text { Fontes DMA et al. }\end{array}$ & $\begin{array}{l}\text { A produção de software por enfermeiros para utilização na assistência ao } \\
\text { paciente. }\end{array}$ & 2016 \\
\hline 2 & $\begin{array}{l}\text { Freitas LCM, Guedes MTS, } \\
\text { Santiago LC. }\end{array}$ & $\begin{array}{l}\text { Proposta de um software-protótipo para uso na assistência a pacientes com } \\
\text { cateter central de inserção periférica (PICC). }\end{array}$ & 2017 \\
\hline 3 & $\begin{array}{l}\text { Junior MGS, Araújo EC, Moraes } \\
\text { CRS et al. }\end{array}$ & $\begin{array}{l}\text { Software para Sistematização da Assistência de Enfermagem em unidade de } \\
\text { internação hospitalar. }\end{array}$ & 2018 \\
\hline 4 & Lima CSP, Barbosa SFF. & $\begin{array}{l}\text { Aplicativos móveis em saúde: caracterização da produção científica da } \\
\text { enfermagem brasileira. }\end{array}$ & 2019 \\
\hline 5 & $\begin{array}{l}\text { Milão LF, Vieira TW, Santos ND et } \\
\text { al. }\end{array}$ & $\begin{array}{l}\text { Integração de tecnologias digitais no ensino de enfermagem: criação de um } \\
\text { caso clínico sobre úlceras por pressão com o software SIACC. }\end{array}$ & 2017 \\
\hline 6 & $\begin{array}{l}\text { Monteiro APTAV, Curado M, } \\
\text { Queiros P. }\end{array}$ & Biotecnologia: revolução digital e conhecimento estético em enfermagem. & 2017 \\
\hline 7 & $\begin{array}{l}\text { Oliveira DAL, Silva MST, Silva } \\
\text { RKS et al. }\end{array}$ & Enfermagem e tecnovigilância na assistência segura. & 2019 \\
\hline 8 & $\begin{array}{l}\text { Pissaia LF, Rehfeldt MJH, Costa } \\
\text { AEK et al. }\end{array}$ & $\begin{array}{l}\text { Os processos que unem tecnologias e ensino na compreensão da } \\
\text { Sistematização da Assistência de Enfermagem e do software Tasy. }\end{array}$ & 2020 \\
\hline 9 & $\begin{array}{l}\text { Salvador PTCO, Gomes ATL, } \\
\text { Rodrigues CCFM et al. }\end{array}$ & $\begin{array}{l}\text { Uso do Software Iramuteq nas pesquisas brasileiras da área da saúde: uma } \\
\text { scoping review. }\end{array}$ & 2018 \\
\hline 10 & $\begin{array}{l}\text { Siqueira VRB, Vasconcelos WPC, } \\
\text { Santos GM et al. }\end{array}$ & $\begin{array}{l}\text { Contribuições da Tecnologia para Assistência de Enfermagem da Unidade de } \\
\text { Terapia Intensiva. }\end{array}$ & 2019 \\
\hline
\end{tabular}

Fonte: Autores (2021).

Ao se analisar os títulos das produções no Quadro 2 pode-se notar que a maioria das produções científicas selecionadas (artigos 1, 2, 3, 5, 9, 10) tratam da temática envolvendo a aplicação de determinadas tecnologias e softwares no campo da assistência de enfermagem, enquanto as demais constituem-se de estudos de literatura sobre essa temática. 
Quadro 3 - Distribuição dos artigos científicos por base de dados, periódico, tipo de estudo e objetivo.

\begin{tabular}{|c|c|c|c|c|}
\hline $\begin{array}{l}\mathbf{N}^{\circ} \text { do } \\
\text { Artigo }\end{array}$ & $\begin{array}{ll}\text { Base de } \\
\text { dados }\end{array}$ & Periódico & Tipo de Estudo & Objetivo \\
\hline 1 & $\begin{array}{l}\text { Periódicos } \\
\text { CAPES }\end{array}$ & $\begin{array}{l}\text { Revista de Pesquisa } \\
\text { Cuidado é } \\
\text { Fundamental Online }\end{array}$ & $\begin{array}{l}\text { Estudo } \\
\text { bibliométrico }\end{array}$ & $\begin{array}{l}\text { Analisar a produção científica referente às estratégias } \\
\text { utilizadas para produção de software por enfermeiros para a } \\
\text { aplicação na assistência ao paciente através de uma revisão } \\
\text { da literatura publicada entre } 1985 \text { e } 2013 \text {, e sintetizar os } \\
\text { achados. }\end{array}$ \\
\hline 2 & LILACS & $\begin{array}{l}\text { Revista Online de } \\
\text { Pesquisa Cuidado é } \\
\text { Fundamental }\end{array}$ & Estudo Qualitativo & $\begin{array}{l}\text { Propor um software-protótipo como estratégia para melhorar } \\
\text { o processo de comunicação e os registros relativos ao } \\
\text { cuidado de enfermagem a pacientes com cateter central de } \\
\text { inserção periférica (PICC) e descrever os passos para a } \\
\text { construção de um protótipo de software. }\end{array}$ \\
\hline 3 & $\begin{array}{l}\text { Periódicos } \\
\text { CAPES }\end{array}$ & $\begin{array}{l}\text { Revista Brasileira de } \\
\text { Enfermagem }\end{array}$ & Estudo Qualitativo & $\begin{array}{l}\text { Descrever o desenvolvimento de um software protótipo para } \\
\text { aplicar o Processo de Enfermagem em unidades de } \\
\text { clínica médica de um hospital geral e avaliar sua utilidade. }\end{array}$ \\
\hline 4 & $\begin{array}{l}\text { Periódicos } \\
\text { CAPES }\end{array}$ & $\begin{array}{l}\text { Revista Eletrônica de } \\
\text { Enfermagem }\end{array}$ & $\begin{array}{l}\text { Estudo } \\
\text { bibliométrico }\end{array}$ & $\begin{array}{l}\text { Identificar o desenvolvimento de aplicativos móveis em } \\
\text { saúde na produção científica brasileira de dissertações e teses } \\
\text { de enfermagem. }\end{array}$ \\
\hline 5 & LILACS & $\begin{array}{l}\text { Revista Eletrônica de } \\
\text { Comunicação } \\
\text { Informação e } \\
\text { Inovação em Saúde }\end{array}$ & Estudo Qualitativo & $\begin{array}{l}\text { Apresentar o desenvolvimento de simulação de um caso } \\
\text { clínico por meio do software SIACC (Sistema } \\
\text { Interdisciplinar de Análise de Casos Clínicos) para auxiliar } \\
\text { o estudo de fundamentos de enfermagem. }\end{array}$ \\
\hline 6 & $\begin{array}{l}\text { Periódicos } \\
\text { CAPES }\end{array}$ & $\begin{array}{l}\text { Revista } \\
\text { Enfermagem } \\
\text { Referência }\end{array}$ & Estudo Qualitativo & $\begin{array}{l}\text { Analisar reflexivamente as novas possibilidades de } \\
\text { pensamento e conhecimento } \\
\text { em enfermagens trazidas pelas biotecnologias e revolução } \\
\text { digital, a partir da perspectiva de Bárbara Carper sobre o } \\
\text { conhecimento estético em enfermagem. }\end{array}$ \\
\hline 7 & $\begin{array}{l}\text { Periódicos } \\
\text { CAPES }\end{array}$ & $\begin{array}{l}\text { Revista Visão em } \\
\text { Debate }\end{array}$ & $\begin{array}{l}\text { Estudo } \\
\text { bibliométrico }\end{array}$ & $\begin{array}{l}\text { Elucidar as ações de prevenção de eventos adversos na } \\
\text { assistência de enfermagem vinculado a implementação do } \\
\text { monitoramento da tecnovigilância. }\end{array}$ \\
\hline 8 & $\begin{array}{l}\text { Periódicos } \\
\text { CAPES }\end{array}$ & Revista SUSTINERE & $\begin{array}{l}\text { Estudo Qualitativo, } \\
\text { descritivo e } \\
\text { exploratório }\end{array}$ & $\begin{array}{l}\text { Este estudo buscou averiguar os conhecimentos prévios de } \\
\text { estudantes de enfermagem sobre a Sistematização da } \\
\text { Assistência de Enfermagem e sobre o software Tasy. }\end{array}$ \\
\hline 9 & $\begin{array}{l}\text { Periódicos } \\
\text { CAPES }\end{array}$ & $\begin{array}{l}\text { Revista Brasileira em } \\
\text { Promoção da Saúde }\end{array}$ & Estudo Qualitativo & $\begin{array}{l}\text { Identificar o uso do Interface de } R \text { pour les Analyses } \\
\text { Multidimensionnelles de Textes et de Questionnaires } \\
\text { (IRAMUTEQ), nas pesquisas qualitativas produzidas no } \\
\text { âmbito dos programas de pós-graduação da área da saúde no } \\
\text { Brasil. }\end{array}$ \\
\hline 10 & $\begin{array}{l}\text { Periódicos } \\
\text { CAPES }\end{array}$ & Revista SUSTINERE & $\begin{array}{l}\text { Estudo descritivo } \\
\text { de corte transversal }\end{array}$ & $\begin{array}{l}\text { Investigar quais as contribuições da evolução tecnológica } \\
\text { sobre a ótica dos enfermeiros dentro da Unidade de Terapia } \\
\text { Intensiva (UTI), analisando as vantagens e desvantagens do } \\
\text { uso da mesma na assistência ao paciente. }\end{array}$ \\
\hline
\end{tabular}

Fonte: Periódicos CAPES e LILACS.

Ao se observar o terceiro quadro, fica evidente que os principais objetivos das produções científicas supracitadas são voltados para a análise das contribuições e os impactos que tecnologias como o uso de softwares traz para o processo de enfermagem dentro da prática assistencial, bem como a percepção de usuários e profissionais da saúde acerca dessa tendência na área da saúde, o que indica a importância de se buscar observar e compreender avanços e descobertas envolvidos nessa temática.

Assim, conforme Freitas, Guedes e Santiago (2017), ao serem aderidas na prática do profissional de saúde, essas novas tecnologias exigem cada vez mais a aprender a lidar com elas, assim como sintetizar estratégias fundamentais para sua utilização 
no panorama hospitalar, haja vista que o enfermeiro se depara com novos desafios perante as transformações tecnológicas e à sua inserção no mundo do trabalho.

Com a tecnologia fortemente incorporada ao meio social e os avanços tecnológicos no âmbito da saúde, os profissionais de enfermagem são obrigados a passar por um processo adaptativo mesclando teoria, procedimentos técnicos e tecnologia, o que resulta em inúmeros benefícios ao paciente, como aprimorar o cuidado e reduzir o tempo de tratamento e internação (Oliveira et al., 2019; Pissaia et al., 2020; Siqueira et al., 2019).

O uso de novas tecnologias na saúde e seus avanços promoveram uma acentuada eficácia nos cuidados da saúde. Para diversos desenvolvedores de apps, os aplicativos móveis têm muitos propósitos, como o gerenciamento da saúde, o monitoramento de doenças, melhorias na adesão de tratamentos e reabilitação de doenças (Lima \& Barbosa, 2019; Monteiro, Curado, \& Queiros, 2017).

Dessa forma, é válido ressaltar que a enfermagem e suas intervenções em equipe são beneficiadas por essas tecnologias, podendo chegar a níveis de excelência, mediante a aplicação desses sistemas, os quais devem ser elementos integradores dentro do contexto da assistência de enfermagem, sendo uma ferramenta de apoio na obtenção de novas informações e conhecimentos para as futuras gerações (Santos, 2010; Siqueira et al., 2019).

\section{Considerações Finais}

Os achados permitem evidenciar a excelente contribuição ímpar e complementar que as tecnologias têm a oferecer para a enfermagem, visto que diversos achados mostram os resultados positivos no âmbito da assistência, principalmente no quesito de facilitar o trabalho em equipe do enfermeiro, o qual muitas vezes passa por rotinas desgastantes. Outrossim, os enfermeiros reconhecem a importância dessas tecnologias tanto na parte gerencial como educacional, apesar de terem poucos estudos que abordem sobre essa temática. Desse modo, observa-se a necessidade de mais estudos na área tecnológica em conjunto com a enfermagem para que se possa ter mais avanços e contribuições e, por conseguinte, uma atuação mais segura dos profissionais de enfermagem dentro da prática hospitalar e comunitária.

Por fim, almeja-se que a presente produção possa servir de fomento a outros trabalhos científicos dentro do campo das tecnologias assistenciais em saúde, como os softwares. De outro modo, os autores sugerem que a realização de trabalhos científicos nesse campo se faça mais abundante, para que se tenha um maior quantitativo de estudos a serem observados e avaliados conforme suas influências, vantagens, implicações e impactos na vida de profissionais e usuários do sistema de saúde, no que se refere à aplicação de tecnologias na assistência em saúde.

\section{Referências}

Acauan, L. V., Abrantes, C. V., Stipp, M. A. C., Trotte, L. A. C., Paes, G. O., \& Queiroz, A. B. A. (2020). Utilização do software iramuteq para análise dos dados qualitativos na enfermagem: um ensaio reflexivo. Revista mineira de enfermagem, 24, 1-5. https://doi.org/10.5935/1415-2762.20200063.

Alves, A. G., Cesar, F. C. R., Martins, C. A., Ribeiro, L. C. M., Oliveira, L. M. A. C., Barbosa, M. A., \& Moraes, K. L. (2020). Tecnologia de informação e comunicação no ensino de enfermagem. Acta Paulista de Enfermagem, 33, 1-8. http://dx.doi.org/10.37689/acta-ape/2020ao01385.

Carlantonio, L. F. M., Freitas, L. C. M., Fontes, D. M. A., Silva, C. F. B., \& Santiago, L. C. (2016). A produção de software por enfermeiros para utilização na assistência ao paciente. Revista de pesquisa cuidado é fundamental online, 8(2), 4121-4130. https://dx.doi.org/10.9789/2175-5361.2016.v8i2.4121-4130.

Costa, M. K., Silva, L. R. F. M. S., Nunes, B. S., Melo, R. F., Rocha, T. N. A., \& Estevam, A. S. (2019). As tecnologias da informação e comunicação no âmbito da enfermagem. Revista científica de enfermagem, 9(27), 108-116. https://www.recien.com.br/index.php/Recien/article/view/308.

Farias, E. B. P., Gatto, D. D. O., Romero, M., Moura, M. L. A. O., \& Sassi, R. J. (2021). Processo de Enfermagem Apoiado por Sistema Especialista na Aplicação das Escalas de Glasgow e Braden em um Hospital Público Brasileiro. Revista de gestão e tecnologia, 11, 1-16. https://doi.org/10.22279/navus.2021.v11.p0116.1397.

Freitas, L. C. M., Guedes, M. T. S., \& Santiago, L. C. (2017). Proposta de um software-protótipo para uso na assistência a pacientes com cateter central de inserção periférica (PICC). Revista online de pesquisa cuidado é fundamental, 9(2), 536-544. https://dx.doi.org/10.9789/2175-5361.2017.v9i2.536-544. 
Friedrich, P. (2019). Software para passagem de plantão de enfermagem na unidade de tratamento intensivo. Dissertação (mestrado) - Universidade do Vale do Rio dos Sinos, Programa de Pós-Graduação em Enfermagem http://www.repositorio.jesuita.org.br/handle/UNISINOS/9010.

Goncalves, D. F. (2020). A apresentação da importância da enfermagem para os futuros profissionais da área para um sus melhor. $14^{\circ}$ Congresso Internacional da Rede Unida. Saúde em Redes, 6(3). ISSN 2446-4813.

Junior, M. G. S., Araújo, E. C., Moraes, C. R. S., \& Gonçalves, L. H. T. (2018). Software para Sistematizacao da Assistencia de Enfermagem em unidade de internacao hospitalar. Revista brasileira de enfermagem, 71(5), 2425-2431. http://dx.doi.org/10.1590/0034-7167-2016-0386.

Lima, C. S. P., \& Barbosa, S. F. F. (2019). Aplicativos móveis em saúde: caracterização da produção científica da enfermagem brasileira. Revista eletrônica de enfermagem, 21, 1-11. https://doi.org/10.5216/ree.v21.53278.

Machado, A. L. G., \& Vieira, N. F. C. (2020). Uso do software webQDA na pesquisa qualitativa em enfermagem: relato de experiência. Revista brasileira de enfermagem,73(3), 1-6. http://dx.doi.org/10.1590/0034-7167-2018-0411.

Milão, L. F., Vieira, T. W., Santos, N. D., Silva, A. P. S. S., \& Flores, C. D. (2017). Integração de tecnologias digitais no ensino de enfermagem: criação de um caso clínico sobre úlceras por pressão com o software SIACC. Revista eletrônica de comunicação, informação e inovação em saúde, 11(1). https://dx.doi.org/10.29397/reciis.v11i1.1189.

Monteiro, A. P. T. A. V., Curado, M., \& Queirós, P. (2017). Biotecnologia: revolução digital e conhecimento estético em enfermagem. Revista de enfermagem referência, 4(13), 139-146. https://doi.org/10.12707/RIV17020.

Mota, N. P., Vieira, C. M. A., Nascimento, M. N. R., Bezerra, A. M., Quirino, G. S., \& Félix, N. D. C. (2019). Aplicativo móvel para ensino da Classificação Internacional para a Prática de Enfermagem. Revista Brasileira de Enfermagem, 72(4), 1077-1084. http://dx.doi.org/10.1590/0034-7167-2018-0751.

Oliveira, D. A. L., Silva, M. S. T., Silva, R. K. S., Cintra, T. D., \& Medeiros, R. R. (2019). Enfermagem e tecnovigilância na assistência segura. Vigilância Sanitária em debate: sociedade, ciência e tecnologia, 7(1), 48-52. https://doi.org/10.22239/2317-269x.001171.

Pereira, S. A., Shitsuka, D. M., Parreira, F. J., \& Shitsuka, R. (2018). Metodologia da pesquisa científica (1a ed.). Santa Maria: NTE. https://www.ufsm.br/app/uploads/sites/358/2019/02/Metodologia-da-Pesquisa-Cientifica_final.pdf.

Pissaia, L. F., Rehfeldt, M. J. H., Costa, A. E. K., Moreschi, C., \& Monteiro, S. (2020). Os processos que unem tecnologias e ensino na compreensão da Sistematização da Assistência de Enfermagem e do software Tasy. Revista Sustinere, 8(2), 315-337. http://dx.doi.org/10.12957/sust inere.2020.39539.

Salvador, P. T. C.B O., Gomes, A. T. L., Rodrigues, C. C. F. M., Chiavone, F. B. T., Alves, K. Y. A., Bezerril, M. S., \& Santos, V. E. P. (2018). Uso do software iramuteq nas pesquisas brasileiras da área da saúde: uma scoping review. Revista brasileira em promoção da saúde, 31, 1-9. http://dx.doi.org/10.5020/18061230.2018.8645.

Santos, S. V., Ramos, F. R. S., Costa, R., \& Batalha, L. M. C. (2020). Avaliação da qualidade de um software para prevenção de lesões de pele em recémnascidos. Revista Latino-Americana de Enfermagem, 28, 1-12. http://dx.doi.org/10.1590/1518-8345.3711.3352.

Siqueira, V. R. B., Vasconcelos, W. P. C., Santos, G. M., \& Oliveira, G. K. S. (2019). Contribuições da Tecnologia para Assistência de Enfermagem da Unidade de Terapia Intensiva. Revista Sustinere, 7(1), 19-31. http://dx.doi.org/10.12957/sustinere.2019.40086.

Torres, G. M. C., Figueiredo, I. D. T., Candido, J. A. B., Morais, A. P. P., \& Almeida, M. I. (2018). O uso de tecnologias leves no cuidado ao hipertenso na Estratégia Saúde da Família. Escola Anna Nery, 22(3). https://doi.org/10.1590/2177-9465-ean-2017-0169. 